\title{
Neutron Diffraction Strain Measurement During TIG Welding
}

\author{
U. Reisgen ${ }^{1}$, R. Sharma ${ }^{1}$, J. von der Heydt ${ }^{1}$ * \\ ${ }^{1}$ ISF - Welding and Joining Institute, RWTH Aachen University, Pontstraße 49, 52062 Aachen, \\ Germany, vonderheydt@isf.rwth-aachen.de, ${ }^{\text {* }}$ corresponding author
}

Keywords: Residual Stresses, Thermal Strain, Welding, Hot Cracking

\begin{abstract}
Development of hot cracks during welding of austenitic materials is a challenge which must be coped with when a suitable welding method is to be chosen. Boundary conditions about hot crack formation are not sufficiently known yet. One factor is the state of strain during welding. Therefore, strain is determined via neutron diffractometry next to the fusion line during the welding process. This evaluation allows to draw conclusions about the influence of the state of thermal strain on the hot crack formation.
\end{abstract}

\section{Introduction}

During the welding processing of metals, gradients of temperature and stresses cause the development of residual stresses which remain in the work-piece after solidification and cooling. These residual stresses are, in part, relieved by plastic deformation during the cooling process. The result is distortion of the component. The stresses which are, afterwards, remaining in the component can be measured using different methods, such as X-ray diffractometry or strain gauges. The strain state and the strain rate in the vicinity of the welded seam directly after the solidification play a decisive part in the development of hot cracks, a defect, which occurs particularly in welding of face-centered cubic materials. Hot cracks occur in the welded seam as so-called solidification cracks, they may also occur in the region of the fusion line and/or in the heat affected zone (liquation cracks). Fig. 1 depicts an example of widened grain boundaries which is said to be the preliminary step for liquation cracks. These phenomena are due to the melting of grain boundary deposits during the welding process and the co-occuring strains at very high temperature.

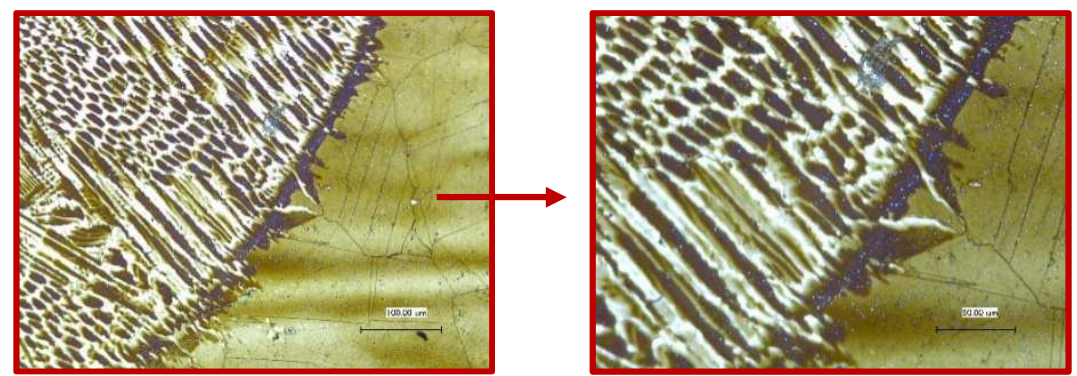

Figure 1: Grain boundary melting, multipass welding of alloy 617

The state of residual stress which remains in the material after cooling may enhance the already existing defect, it is, however, not responsible for its development.

In order to determine the strain which is responsible for the development of this effect, application of standard methods for strain determination, such as X-ray diffractometry or strain gauges is inadequate on the material surface or it is not possible at all. Neutron diffractometry is a possibility of measuring the relevant strain in the region of high temperature, also in the material depth. The advantage of this method is that the running welding process remains uninfluenced during the measurement, thus allowing measurement of the strain in the solidified, still hot weld material and/or next to it, under realistic boundary conditions. 


\section{Measuring Method and Theoretical Fundamentals for the Strain Development during Welding}

Neutron diffractometry allows the non-destructive measurement of elastic strains in steel with a thickness of up to $20 \mathrm{~mm}$. This method is, therefore, particularly suitable for the measurement of the strains which are responsible for hot-crack formation. A neutron ray is guided onto a work-piece and diffracted at the lattice structure of the material. From this and other boundary conditions which are a result from the used material, the following is established:

$n \cdot \lambda=2 d_{h k l} \cdot \sin \Theta \quad$ (Bragg equation)

Equation 1

Here, $n$ stands for the order of diffraction, $\lambda$ stands for the wavelength, $d_{h k l}$ is the lattice plane spacing of the two considered lattice orientations and $\Theta$ is the diffraction angle. The measurement of elastic states of strain, as scheduled for this experiment, is carried out via differential determination of the diffraction angle between a reference (here: cold weld specimen) and the measuring volume which has to be examined during the welding processing. The following connection was found:

$\varepsilon=\frac{d-d_{0}}{d_{0}}=\frac{\sin \theta_{0}}{\sin \theta}-1$

Equation 2

In this way, however, the strain in one spatial direction is determined. For the determination of the state of stress in the material, measurement of the strains in all three main strain directions must be made. Within the scope of this experiment, only the determination of the strains in two spatial directions has, for process and equipment reasons, been possible. It has been possible to determine the diffraction angles longitudinally and also transversally to the weld direction.

The measurements which are specified here have been made with the STRESSPEC instrument at the FRMII (Technical University of Munich, Germany).

\section{Experimental set-up and test material}

Due to the high process stability and the good manageability, TIG welding without additional filler wire has been chosen for this in-situ experiment. Here, an arc develops between a non-consumable electrode and the work-piece. In order to keep the boundary conditions of this diffraction experiment as simple as possible, filler wire is not added into the process. Solely a melting of the base material takes place.

In order to ensure measuring time with a sufficiently long duration, a stationary welding torch is installed on the sample table and the specimen is guided underneath the torch. Thus, the relative position of the torch to the measuring point is always maintained and sufficient time for measuring is ensured. For this approach, one specimen each per measuring point and spatial direction is required. Fig. 2 depicts the network of the measuring points which are to be considered 


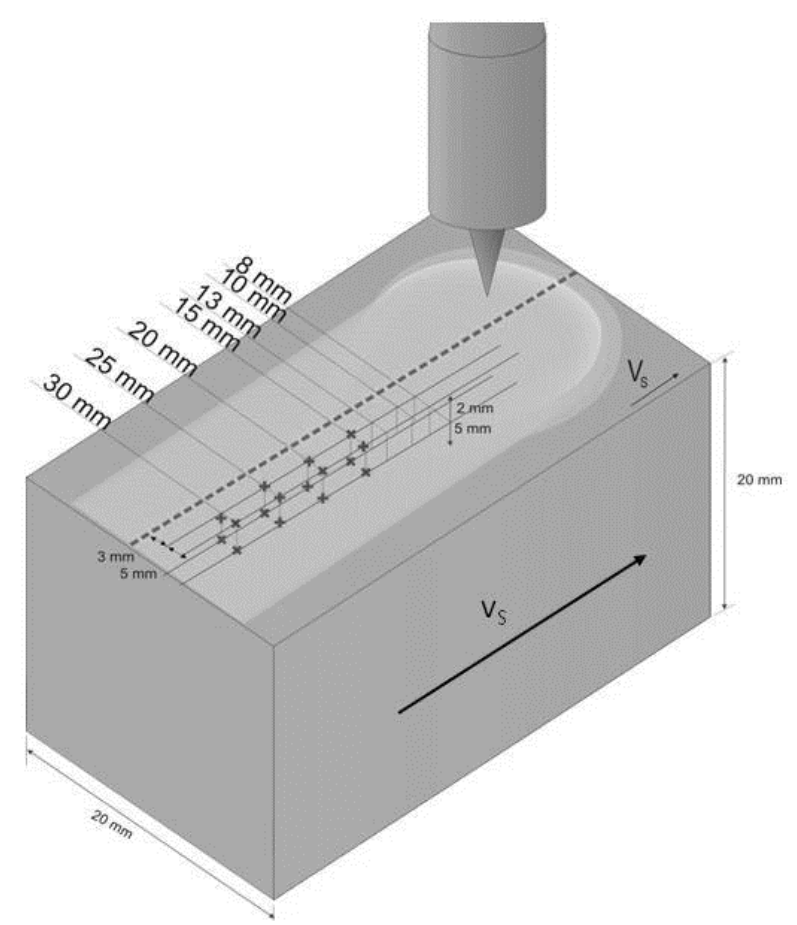

Figure 2: Position of the measuring points, relative to torch position

The test material is the austenitic steel 1.4435. Due to its lattice structure, this steel can show a tendency to hot cracking during the welding process. The used material has the shape of a rod and has the dimensions $20 \times 20 \times 300 \mathrm{~mm}^{3}$.

In a first step before the experiment can start the aquisition of the diffraction patterns during the welding experiment has been carried out. Therefore the neutron gauge volume was positioned in constant distance to the welding heat source after Fig. 2. A longitudinal section of the weld centerline has been prepared in advance to estimate the geometry of the weld pool. In conventional macrosections of welds, the actual outline of the weld pool is not visible but only the maximum of the molten area. To face this deficiency, the weld pool was blown out using a high pressure gas surge while the welding process was simultaneously aborted. In Fig. 3 and Fig. 4, the position of the measuring points are highlighted. Only the measuring points in a depth of $2 \mathrm{~mm}$ from the top of the sample and a distance of $3 \mathrm{~mm}$ to the centerline are positioned within the solidified weld metal, All other measuring points are located in the heat affected zone.

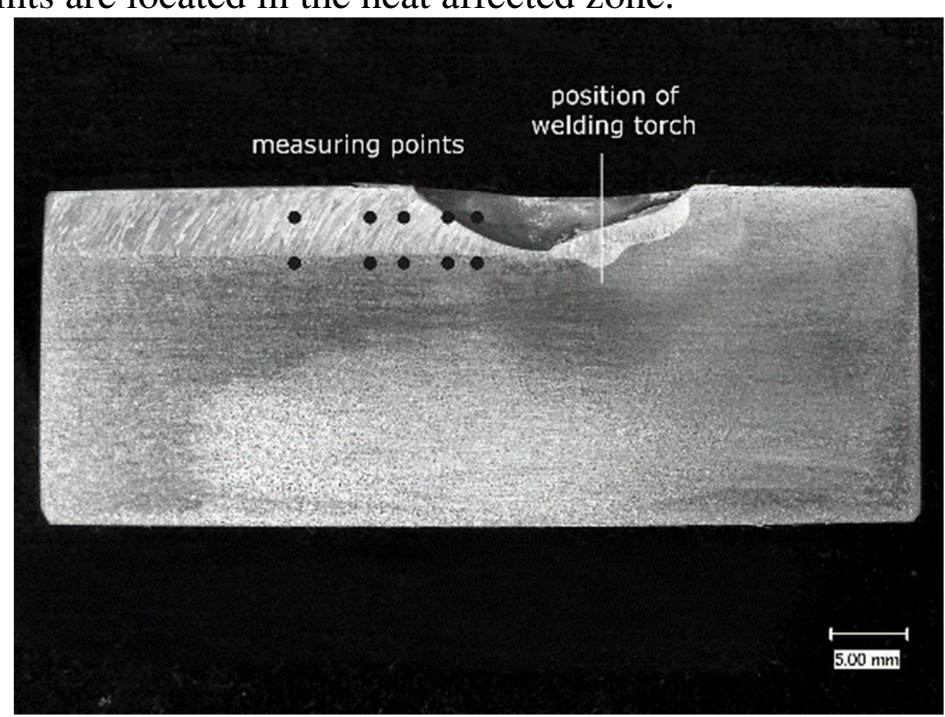

Figure 3: Position of the measuring points relative to the welding torch in a longitudinal section of a welded sample, blown out weld pool 


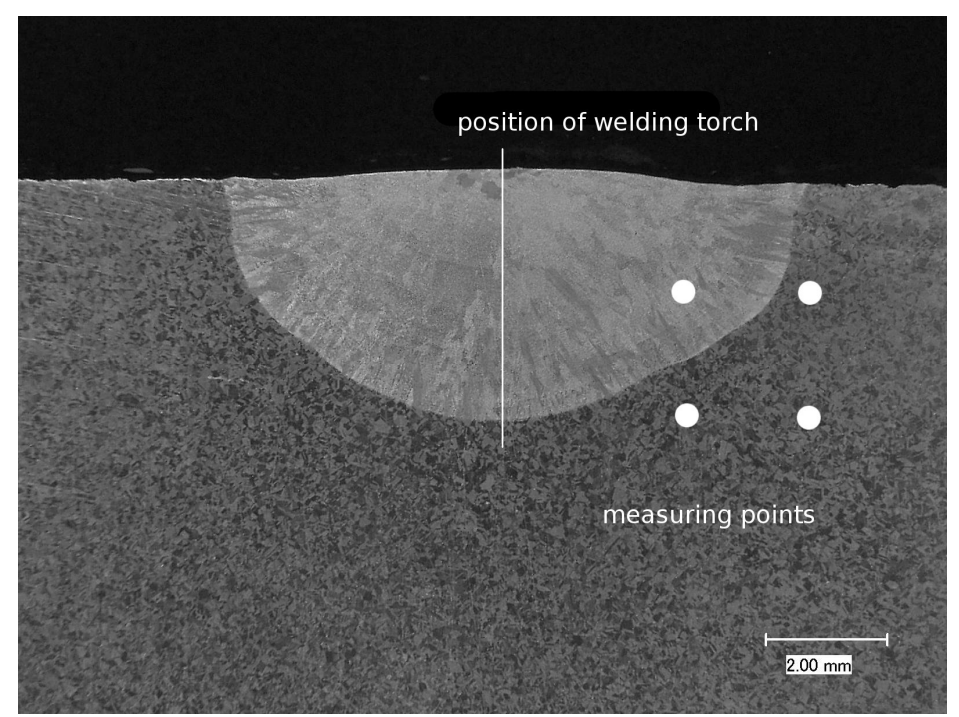

Figure 4: Position of the measuring points relative to the welding torch in cross section

The diffraction angle is composed of a weld process-induced part and a diffraction part which is induced by the thermal expansion of the material. It is necessary to eliminate the latter in order to achieve statements about the strains which are relevant for hot crack development.

Therefore, an additional experiment is carried out for the determination of the thermal expansion. A cylinder-shaped sample was placed into a vacuum furnace which was mounted onto the diffraction sample table. Afterwards, a heating cycle with hold points in steps of $50{ }^{\circ} \mathrm{C}$ has been conducted. When a particular temperature inside the sample was reached, a diffraction pattern was acquired. In addition, the simulation of the temperature field in the region of the weld seam based on the used weld parameters has been carried out. For the simulation, the ESI Sysweld 2011 software is used. In order to receive simulation results which are as precise as possible, the results are compared with pyrometer measurements on the surface of the specimen. In this way it is possible to assign, also in the depth of the material, a temperature to each measuring point from the diffraction experiment.

\section{Results of the Experiment}

The lattice parameters $a_{311}$ at the respective measuring points are depicted for one spatial measuring direction in the diagrams in Fig. 5.

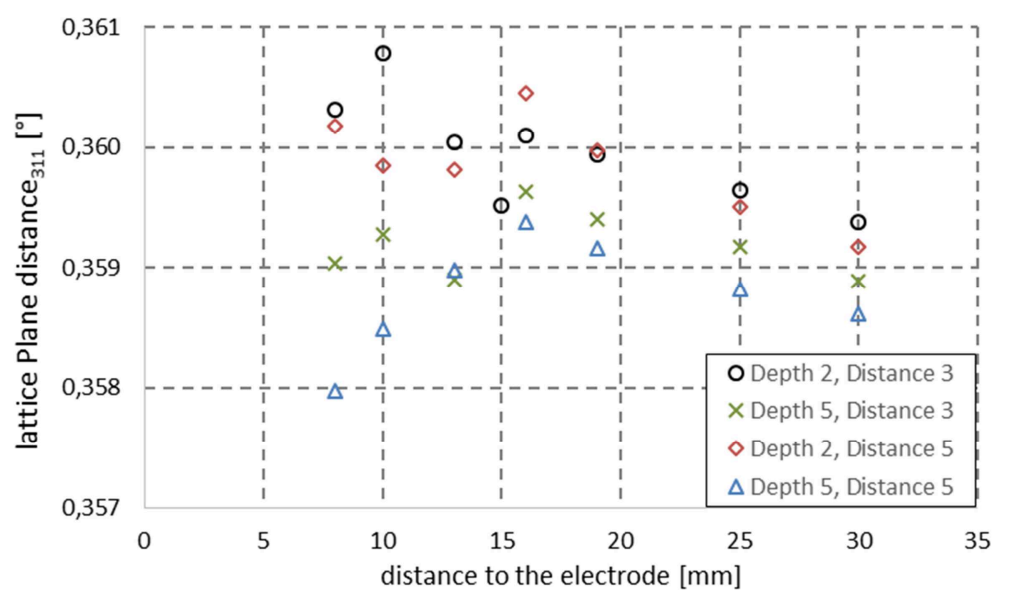

Figure 5:Spatial distribution of measured lattice plane distance in one spatial direction

When considering the measured results, the sometimes substantial fluctuations of the lattice plane distance at the same measuring point are attracting attention. There are several reasons for this effect. One reason is the complex measurement set-up and the various influences on the measuring precision which are a result thereof. A further reason may be the crystalline structure of the used 
material. Since a different specimen has been used for each measurement, it is possible that changes in the thermal field between the different specimens led to fluctuations of diffraction angles.

As already specified above, the thermal expansion of the measured diffraction angles is determined by the consolidation of the measurements of the purely temperature-induced diffraction angles in the furnace experiment and the results of the simulation of the welding-induced temperature field. From this diffraction angle, now the state of strain in the respective spatial directions can be determined by means of comparison with a non-loaded specimen. As already mentioned above it has, due to the orientation of the detector to the specimen, not been possible to determine the strains in the third spatial direction. Therefore, only the difference of the two principal stresses in the measured normal stress directions was calculated:

$\delta \sigma=\sigma_{y}-\sigma_{x}=\frac{E_{h k l}}{1+v_{h k l}}\left(\varepsilon_{y}-\varepsilon_{x}\right)$

Equation 3

The values of elastic modulus and poisson ratio are temperature dependent and were calculated using the software JmatPro. Because the 311-reflex has been observed during the experiment, there is only a slight error due to the use of macroscopic elastic constants.

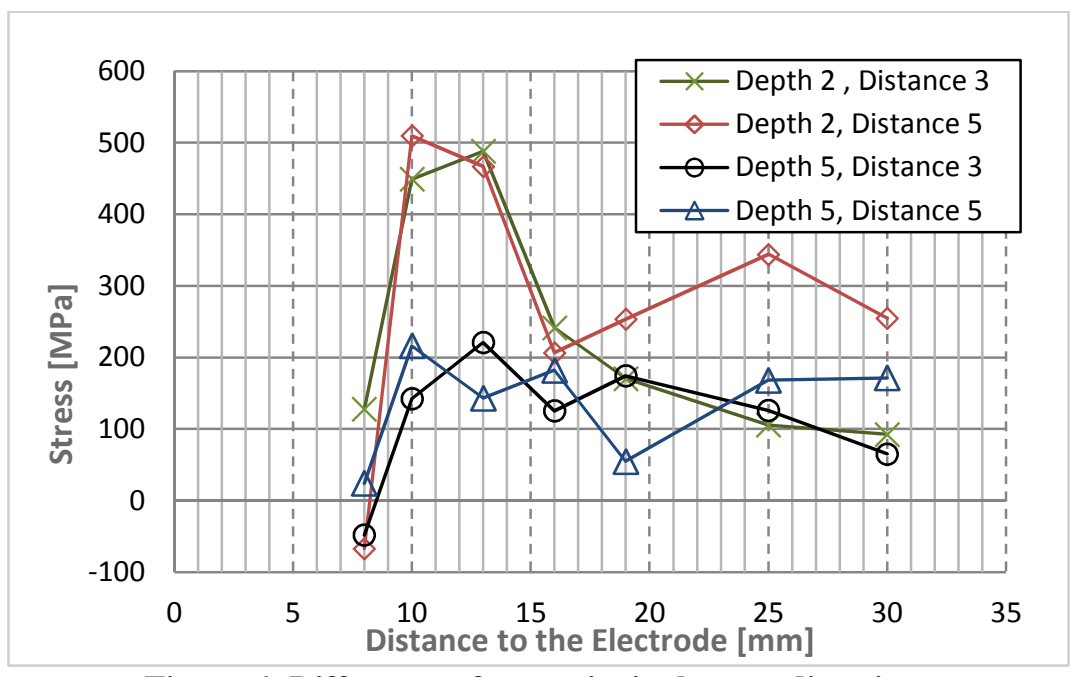

Figure 6: Difference of two principal stress directions

The determined difference in stress is depicted in Fig. 6. The curve of the stresses shows that in a depth of $2 \mathrm{~mm}$, i.e. in the vicinity of the molten pool, a tensile stress magnitude has been reached. Based on this curve one can assume that both measurement points are positioned in the weld metal which has just solidified and that here the expected tensile stresses which are due to volume shrinkage are developing. The tendential curve of the stresses is thus realistic. The magnitude of the calculated stresses remains, however, subject to discussion. At the prevailing temperature the material should rather not show such high strength.

\section{Evaluation of the Results and Extended Diffraction Experiment in SA Welding}

The evaluation of the results which have been gained so far and the comparison with technical literature shows that the tendential curve of the stresses which have been approximately calculated from the results is realistic [5].

If the magnitude of the diffraction angle differences (Fig. 6) is considered, it becomes clear that the exact determination of the temperature-induced diffraction proportion is essential for the exact determination of the state of strain. For this reason, the simulation of the temperature on the basis of temperature measurement solely on the material surface is not sufficient. For a more detailed interpretation of the results, the measurement must also be carried out in the depth of the material. Moreover, measurement of the third spatial direction has not been possible. Therefore, only the approximate calculation of the state of stress during welding has been possible. 
Despite the results which are still pending, a further in-situ diffraction experiment is carried out. In contrast to previous experiments, SA welding has been chosen as the welding method. Other than TIG welding, SA welding is characterised by large molten pools which result thus in a higher thermal and mechanical load of the material in the region of the considered measuring points. Furthermore the lower temperature gradient results in a reduced measurement error. Fig. 7 and Fig. 8 show, exemplarily, a photography of a blown-out SA weld seam and also a macro-section of a weld bead in order to allow a comparison with TIG welding (refer also to Figure 3 and Figure 4).

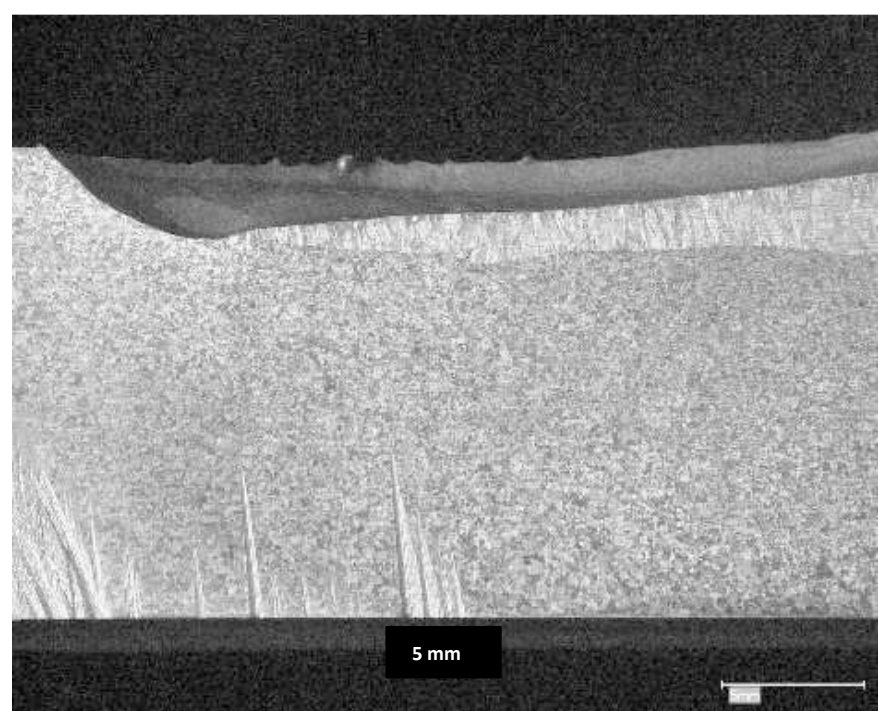

Figure 7: Blown-out weld seam of a SA welding

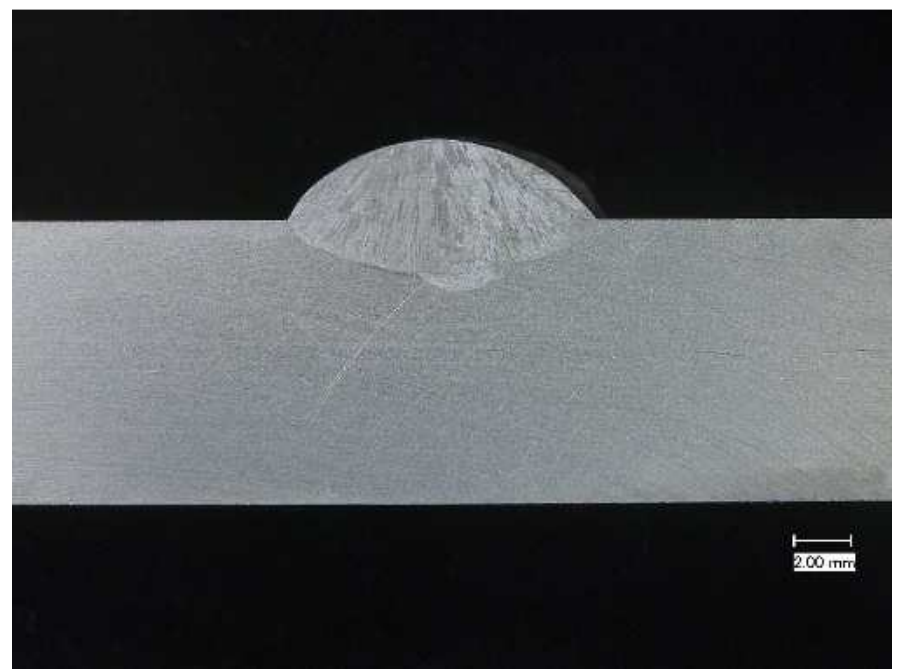

Figure 8: Macro-section of a SA welded seam

The consideration of a further welding method with larger molten pools in the in-situ experiment shows that there is another possibility for the analysis of the results from the experiments. It is, thus, possible to compare the determined strain in the two measured spatial directions, under consideration of the process-specific characteristics. Through the elimination of the thermal diffraction proportion from the results (furnace experiment and simulation of the respective temperature) a direct comparison of the strain at analogous measuring points should be possible and thus allow statements about the process-specific strain-development during welding.

\section{Summary}

The results of the measurements and the stresses which are derived thereof show that it is basically possible to carry out this kind of experiment. The achieved results after the evaluation of the strain 
are, basically, corresponding with the results from other experiments, referred to in technical literature.

Further tests shall serve the purpose of transferring the measurement methods to SA welding. Due to the relatively large molten pools in this welding method it is expected that the states of strain will be pronounced even more clearly. The transmission is, however, impeded by the application of a filler wire and the connected, more complicated boundary conditions, compared to TIG melting beads.

The authors gratefully acknowledge the financial support provided by the German research foundation (DFG- RE2755/13-1) to perform the neutron scattering measurements at Heinz MaierLeibnitz Zentrum (MLZ), Garching, Germany.

\section{References:}

[1] Gottstein, G.,,Physikalische Grundlagen der Materialkunde“ 3.ed., Springer Berlin, 2007

[2] Radaj, D.: „Wärmewirkungen des Schweißens” Springer-Verlag Berlin, 1988

[3] Radaj, D.:“ Eigenspannungen und Verzug beim Schweißen - Rechen und Messverfahren“ Fachbuchreihe Schweißtechnik, Bd. 143, DVS Media Düsseldorf, 2002

[4] Reisgen, U., Schleser, M., Mokrov, O.,: „Diagnostic for simulation of submerged arc welding. combination of direct and indirect capture of weld pool geometry and dynamics" Proceedings of Visual-JW 2012, 28-30 November 2012, Osaka, Japan. Vol. 1, pp. 318-319

[5] Reisgen, U., Geffers, C., Sharma, R., von der Heydt, J.: "In-situ measurement of thermal residual stresses during fusion welding",ICRS9 Proceedings, Materials Science Forum Vols. 768769 